Horizons philosophiques

\title{
La conception herdérienne des chants populaires et une invention du goût universel
}

\section{Hiroshi Yoshida}

Volume 13, numéro 2, printemps 2003

Herder (1744-1803) : le clair-obscur

URI : https://id.erudit.org/iderudit/801236ar

DOI : https://doi.org/10.7202/801236ar

Aller au sommaire du numéro

Éditeur(s)

Collège Édouard-Montpetit

ISSN

1181-9227 (imprimé)

1920-2954 (numérique)

Découvrir la revue

Citer cet article

Yoshida, H. (2003). La conception herdérienne des chants populaires et une invention du goût universel. Horizons philosophiques, 13(2), 51-58.

https://doi.org/10.7202/801236ar d'utilisation que vous pouvez consulter en ligne.

https://apropos.erudit.org/fr/usagers/politique-dutilisation/ 


\section{LA CONCEPTION HERDÉRIENNE DES CHANTS POPULAIRES ET UNE INVENTION DU GOÛT UNIVERSEL1}

En général on s'accorde pour dire que l'homme de lettres et philosophe Johann Gottfried von Herder (1744-1803) est le précurseur de l'intérêt musicologique moderne pour les chants populaires. II est également de notoriété publique que sa conception des Volkslieder était intimement liée au développement du nationalisme culturel moderne dans les pays germanophones. Cependant, jusqu'à ce jour, on n'a guère abordé la question des présupposés philosophiques et esthétiques de sa découverte des chants populaires. Cet article vise justement à préciser l'intérêt de Herder pour les chants populaires en tenant compte des problèmes esthétiques et socioculturels communément débattus dans l'Europe du XVIIIle siècle.

\section{L'influence d'Addison sur Herder. La découverte des «gens ordinaires"}

D'entrée de jeu, nous abordons la question des présupposés philosophiques de l'idée des chants populaires : À l'intérieur de quelle tradition humaniste Herder a-t-il imaginé l'idée des Volkslieder?

Herder était parfaitement conscient qu'il n'était nullement le premier à ressentir un vif intérêt pour les chants populaires. Dans la préface du premier livre des Volkslieder (1778), il cite et traduit un certain nombre de remarques antérieures sur les chants populaires, énoncées non seulement par ses compatriotes, mais également par des auteurs étrangers, dont $M$. Luther (1483-1546), J. Agricola (1499/94-1566), M. E. de Montaigne (1533-1592), Ph. Sidney (15541586), J. Milton (1608-1674), J. Addison (1672-1719), Ch. Burney (1726-1814). G. E. Lessing (1729-1781) et H. W. Gerstenberg (17371823). De ce nombre, Herder semble tout particulièrement influencé par les écrits d'Addison sur les chants populaires parus dans le périodique Spectator, chose que l'on peut bien imaginer de ce critique anglais de premier plan au début du XVIIIe siècle. 
"Lorsque je voyageais," énonce Addison dans un article du 21 mai 1711, «je prenais grand plaisir à entendre les chants et les fables transmis de père en fils et qui sont très en vogue parmi les gens ordinaires des pays que j'ai parcourus ${ }^{2}$. Ces chants, croyait-il, "devraient être universellement estimés et approuvés par la masse en vertu de leur disposition particulière à plaire et contenter l'esprit humain, car la nature humaine est la même chez toutes les créatures raisonnables ${ }^{3}$.

Partageant parfaitement la foi d'Addison dans un trait universel de la nature humaine, Herder traduit et incorpore dans ses Volkslieder des passages d'Addison tirés du même numéro du Spectator : "Un chant populaire ordinaire, ravissant un homme ordinaire, ne peut manquer de plaire à de tels lecteurs car ils n'ont pas la compétence d'être divertis par leur préciosité ou leur ignorance ${ }^{4}$.

Même si nous partageons la croyance d'Addison et de Herder que le goût et le jugement des "gens ordinaires" possèdent une validité universelle, où et comment pouvons-nous les trouver? Cela s'exprime, répond Herder (comme Addison d'ailleurs), dans la raison et le jugement d'une vieille ménagère, ou dans une vieille chanson entonnée par un mendiant aveugle. Par conséquent, force est de conclure que le trait essentiel des "gens ordinaires" consiste dans son caractère anonyme et séculaire. Les chants populaires ne manquent jamais de satisfaire et de contenter l'esprit humain, précisément parce qu'ils nous sont parvenus de génération en génération et qu'ils ont été chantés et embellis de longue date par une masse anonyme.

Mais notre tradition des chants populaires meurt à petit feu, comme l'évoque Herder, car aujourd'hui nous avons pour habitude de "danser des menuets français d'une insupportable manière teutonne, et de chanter (...) d'insupportables chansons paillardes et galantes (unerträgliche schöne Zoten und Buhllieder)". Par conséquent, l'intention de Herder de rassembler et de publier des chants populaires allemands était, comme il l'explique dans une lettre à Nicolai (1773), "d'inciter les gens à recueillir oralement chez le peuple le reste des chants populaires (Nationallieder) ${ }^{5}$ \%.

En opposition marquée à la situation désastreuse prévalente dans les pays germanophones, les hommes de lettres et les érudits anglais se mirent à recueillir puis publier des recueils de leurs chants populaires, tandis que les poètes se consacraient à les étudier et à les 
imiter. Dans Von Ähnlichkeit der mittlern englischen und deutschen Dichtkunst (1777), Herder exprimait de la façon suivante son appréciation sans réserve des réalisations inédites des Anglais :

Les Anglais - avec quelle avidité ils ont recueilli, imprimé et réimprimé, utilisé, lu leurs vieux chants et mélodies! Ramsay, Percy et leurs semblables sont accueillis par acclamation, leurs nouveaux poètes Shenstone, Mason, Mallet : pour le moins avec élégance et sans empressement se sont familiarisés à ce style, Dryden, Pope, Addison, Swift, s'y sont appliqués à leur manière 6 .

\section{De Le Cerf à Herder. Bon goût et sentiment naturel}

L'évaluation positive par Herder du goût et du jugement de la "multitude», une des conditions préalables de son vif intérêt pour les chants populaires (Volkslied), doit être perçue comme une répercussion de la nouvelle tendance dans l'esthétique musicale du XVIIIe siècle. Ceci constitue le second point de notre argument.

L'idée quasi démocratique du goût peut être attribuée à un écrivain sur la musique : le jésuite français Jean Laurent Le Cerf de la Viéville (1674-1707). Bien que dans l'ensemble sa théorie esthétique de la musique était tributaire de la tradition française du Criticisme classique, le Traité du bon goût en musique de Le Cerf établissait une attitude extrêmement moderne sur le bon goût en musique. Séparant le public musical en France en trois groupes, soit les sçavants, les connoisseurs et le peuple, Le Cerf se rallia au dernier groupe en raison du fait que son jugement sur la musique découle simplement du "sentiment naturel». "Le peuple est», explique Le Cerf, "la multitude, le grand nombre, qui ne s'est point élevé à des connoissances particulières \& n'a pour guide \& pour garand de ses jugements, que le sentiment naturel 7 ", et il poursuit : "Ce peuple-ci, conduit par la nature, à laquelle il s'abandonne, s'entreprêtant chacun ses lumiéres, se redressant l'un l'autre, \& prononçant selon un sentiment commun \& libre, sera ne vous en déplaise, le grand juge ${ }^{8}$.

Cette nouvelle idée de Le Cerf sur le goût musical se propagea rapidement à travers toute l'Europe chez les auteurs sur la musique et cela malgré le fait que son nom et son livre sombrèrent rapidement dans l'oubli. Le Comparaison de la musique italienne et de la musique françoise de Le Cerf, dans lequel on trouve un chapitre intitulé "Traité du bon goût en musique», fut reproduit de façon anonyme dans la 
seconde édition de l'Histoire de la musique (1721) de Jacques Bonnet-Bourdelot, la première histoire de la musique en français et vraisemblablement le livre de musique le plus lu en l'Europe au XVIIIe siècle. La théorie de Le Cerf obtint une vaste audience en raison de son inclusion dans ce livre d'histoire. En outre, son idée a été plagiée lorsque Nicolas Racot de Grandval publia l'Essai sur le bon goust en musique (1732) qui n'était, comme l'ont récemment révélé des spécialistes, rien d'autre qu'un précis du Traité de Le Cerf. Cependant ce fut cette version piratée par Grandval qui livra l'idée de Le Cerf au public allemand. Le critique musical prussien Friedrich Wilhelm Marpurg (1718-95) traduisit l'Essai de Grandval et l'inclut dans différents volumes du Der critische Musicus an der Spree (1749).

L'introduction par Marpurg de la conception démocratique du goût de Le Cerf constitua un moment décisif dans le développement de l'esthétique musicale allemande. De concert avec Le Cerf et Grandval, Marpurg définit le "bon goût» de la façon suivante : " Le bon goût consiste donc dans un sentiment naturel expurgé de toute règle ${ }^{9}$.

D'où l'idée que le bon goût dans cette acception se trouve généralement chez les gens ordinaires (Volk), la masse (der grosse Haufen) plutôt que chez les intellectuels car ceux-ci respectent et obéissent trop souvent aux règles. Nous pouvons donc conclure : "Troisièmement, la plus belle musique est sans contredit celle qui est goûtée tant par le peuple que par les connaisseurs et les érudits, mais je priserai toujours davantage celle qui est généralement goûtée du peuple ${ }^{10}$ ".

Le bon goût ne pouvait plus être défini à partir de règles établies et d'une connaissance de l'art, tel que le préconisait l'esthétique classique. Dorénavant on attribuait son origine aux jugements indépendants des individus comme dans la philosophie morale kantienne. Sous cet angle, on peut raisonnablement conclure que la conception herdérienne du Volk découlait automatiquement de la nouvelle conception du goût. Dans Adrastea (1803), un de ses derniers textes, Herder résume sa conception en adoptant cette vieille maxime provenant d'une pratique légale romaine : "Volkstimme, Gottesstimme (vox populi, vox Del) $)^{11}$ ».

Lorsque dans Von deutscher Art und Kunst (1773) Herder glorifie les chants populaires d'Ossian, poète celtique légendaire du troisième siècle, il exprime résolument son sentiment anti-clérical et anti-académique : 
Sachez également que plus un peuple est sauvage, c'est-àdire plus il est vivant et libre de ses gestes, (car ce mot "peuple" signifie tout cela, ni plus ni moins!) plus sauvage, c'est-à-dire plus vivants, libres, sensuels, lyriques doivent également être ses chants, si jamais il en a! Plus un peuple est dépourvu d'une mentalité, d'un langage et d'une écriture artistiques ou scientifiques, moins ses chants sont faits pour le papier et rimés en vers non déclamés ${ }^{12}(.$.

Prenant distance d'une manière artificielle de penser et des règles de composition savantes, les poèmes d'Ossian, "chants d'un peuple sans instruction mais voluptueux", sont intrinsèquement émouvants. Herder avait appris d'Addison que les chants populaires "sont une simple copie de la nature, dépourvus entièrement des artifices et des ornements de l'art ${ }^{13}$ ".

Bien qu'il ait examiné avec soin une traduction allemande des poèmes d'Ossian (1768-69) publiée par Michael Denis (1729-1800), Herder condamna farouchement le traducteur en raison du fait qu'il imposa "notre belle forme épique» aux chants d'Ossian ruinant ainsi "le caractère sauvage, doux, festif ou belliqueux de leur rythme, de leurs mélodies, de leurs mesures, c'est-à-dire de l'esprit même de la chanson ${ }^{14}$ ». Ici il ne faut pas négliger le fait que Herder utilise l'adjectif "beau» (schön) dans un sens plutôt négatif. II repoussait absolument les règles établies et inflexibles de versification, le rythme romain ou grec, comme l'hexamètre, qui avait été résolument formulé comme la doctrine classique de la poésie au cours des XVIle et XVIIIe siècles.

\section{L'anticlassicisme de Herder comme expression du sentiment patriotique}

Pour Herder et ses contemporains, le qualificatif "classique" s'appliquait uniquement au néoclassicisme français. Exposé dans les travaux de Nicolas Boileau, Charles de Saint-Evremond, Dominique Bouhours et Charles Batteux, le néoclassicisme français trouva écho chez d'ardents partisans allemands, tels Christian Thomasius et Johann Christoph Gottsched. Considérant l'hégémonie culturelle en Europe vers la fin du XVIIII siècle, il était tout naturel que l'antipathie marquée de Herder envers la doctrine classique dans les arts était intimement liée à sa francophobie. Ceci constitue notre dernier argument. 
La citation suivante, tirée de Von Ähnlichkeit, établit au-delà de tout doute le lien unissant son aversion du Classicisme à son sentiment patriotique : "Maintenant nous devons nous prendre en main, tenir tête, chercher, avant que nous nous trouvions tous formés selon le modèle du Classicisme, à chanter des chants français, à danser des menuets français ou bien à écrire des hexamètres et des odes à la manière de Horace ${ }^{15}$ ".

L'accent patriotique exprimé ici peut être correctement compris dans le contexte du mouvement des Lumières et, bien entendu, ne devrait pas être identifié à un prodrome du nationalisme culturel de l'Allemagne des XIX ${ }^{\ominus}$ et $X X^{\ominus}$ siècles. Mais quoi qu'il en soit, Herder fut le premier à canaliser l'opinion du public allemand vers les liens ethniques unissant les pays "nordiques», en plaidant en faveur de la beauté naturelle et universelle dissimulée dans les chants populaires des Anglais, des Celtes, des Islandais, des Lettons, des Estoniens et des Prussiens ${ }^{16}$. Sous cet angle, la conception herdérienne des chants populaires peut être perçue comme une des expressions les plus annonciatrices et vigoureuses du «romantisme politique», tel que l'observa judicieusement Carl Schmitt'7.

Dans les Alte Volkslieder (1774), sources de publications ultérieures, Herder avait déjà exprimé clairement cette croyance des chants comme expression du romantisme allemand, comme dans le passage suivant :

Le littoral entier de la mer Baltique comporte en partie des peuples dont l'histoire est assurément plus éclairée, comme leur histoire naturelle, la science même de leur langue et manière de penser. Les Wendes, les Slaves, les anciens Prussiens, les Lithuaniens, les Lettons, les Estoniens, même les lointains Frisons, ceux-ci sont tous tenus à l'encontre de nations que nous reconnaissons, en particulier les Islandais, alors les comparera-t-on juste en essayant de considérer leur façon de penser et leur langue ${ }^{18}$ ?

Tout aussi injuste qu'il soit de le qualifier de nationaliste selon l'acception moderne du mot, on ne peut nier que la conception de Herder des chants populaires a non seulement façonné le cadre conceptuel du jugement esthétique des Européens modernes, mais a également orienté leur imagination anthropologique et géopolitique. En outre, un réexamen de la pensée musicale de Herder engendrera 
nécessairement une nouvelle perspective fascinante dans le domaine de l'histoire et de l'esthétique de la musique, nous incitant à élargir nos champs de recherche et, de surcroît, à redéfinir notre discipline même.

\section{Hiroshi Yoshida \\ Stagiaire post-doctoral, Université de Tokyo qyy02630@nifty.ne.jp}

\section{Bibliographie}

\section{Les œuvres de Herder}

Johann Gottfried von Herder. Schriften zur Ästhetik und Literatur 1767-1781 (= Werke in zehn Bänden, Band 2). Francfort-surle-Main, Deutsche Klassiker Verlag, 1993.

- Volkslieder/Übertragungen/Dichtungen (=Werke in zehn Bänden, Band 3). Francfort-sur-le-Main, Deutscher Klassiker Verlag, 1990.

\section{Autres ouvrages cités :}

Joseph Addison, Richard Steele et al. Spectator. Édité par D.F. Bond. 5 volumes. Oxford, Clarendon Press, 1965.

Jean Laurent Le Cerf de la Viéville. "Un Traité du bon goût en Musique", dans Comparaison de la musique italienne et de la musique françoise. Seconde Partie. Bruxelles, François Foppens, 1705.

Friedrich Wilhelm Marpurg. Der critische Musicus an der Spree. Berlin, A. Haude \& J. C. Spener, 1750.

Carl Schmitt, Politische Romantik. Munich/Leipzig, Duncker et Humboldt, 1919.

1. Cet article et les citations ont été traduits de l'anglais ou de l'allemand par Roch Duval. Le texte allemand est reproduit à la fin de l'article.

2. Joseph Addison, Richard Steele et al, Spectator, Édité par D.F. Bond. 5 volumes. Oxford, Clarendon Press, 1965, i, p. 297.

3. Ibid.

4. «Ein gewöhnlicher Volksgesang, an dem sich der gemeine Mann ergötzet, muß jedem Leser gefallen. der nicht durch Unwissenheit oder Ziererei sich jeder Unterhaltung unfähig gemacht hat.» 
5. Johann Gottfried von Herder, Schriften zur Ästhetik und Literatur 1767-1781 (= Werke in zehn Bänden, Band 2), Francfort-sur-le-Main, Deutsche Klassiker Verlag, 1993, p. 1124.

6. Ibid., p. 554; les italiques sont de Herder . «Die Engländer - mit welcher Begierde haben sie ihre alte Gesänge und Melodien gesammelt, gedruckt und wieder gedruckt, genutzt, gelesen! Ramsay, Percy, und ihres Gleichen sind mit Beifall aufgenommen, ihre neuern Dichter Shenstone, Mason, Mallet haben sich, wenigstens schön und müßig, in die Manier hineingearbeitet: Dryden, Pope, Addison, Swift sie nach ihrer Art gebrauchet (...)".

7. Jean Laurent Le Cerf de la Viéville, "Un Traité du bon goût en Musique", dans Comparaison de la musique italienne et de la musique françoise. Seconde Partie. Bruxelles, François Foppens, 1705, p. 319.

8. Ibid., p. 322-323.

9. Friedrich Wilhelm Marpurg, Der critische Musicus an der Spree, Berlin, A. Haude \& J. C. Spener, 1750, p. 111; "Es bestehet also der gute Geschmack in einer durch die Regeln gereinigten natürlichen Empfindung."

10. Ibid., p. 184; “Drittens ist ohne Widerspruch die schönste Musick diejenige, die sowohl vom Volck, als von Kennern und Gelehrten bewundert wird; ja ich würde allezeit diejenige höher schätzen, welche durchgängig von dem ganzen Volcke bewundert wird."

11. Johann Gottfried von Herder, Volkslieder/Übertragungen/Dichtungen (=Werke in zehn Bänden, Band 3), Francfort-sur-le-Main, Deutscher Klassiker Verlag, 1990. SW XXIV, 271, p. 866.

12. Johann Gottiried von Herder, Schriften, p. 452; “Wissen Sie also, daß je wilder, d.i. je lebendiger, je freiwirkender ein Volk ist, (denn mehr heißt dies Wort doch nicht!) desto wilder, d.i. desto lebendiger, freier, sinnlicher, lyrisch handelnder müssen auch, wenn es Lieder hat, seine Lieder sein! Je entferner von künstlicher, wissenschaftlicher Denkart, Sprache und Letternart das Volk ist: desto weniger müssen auch seine Lieder fürs Papier gemacht, und tote Lettern Verse sein(...)"

13. Joseph Addison, op.cit., I, p. 362.

14. Johann Gottfried von Herder, Schriften, p. 448, 463.

15. Ibid.,p. 558; «Nur wir müssen Hand anlegen, aufnehmen, suchen, ehe wir Alle klassich gebildet dastehn, französische Lieder singen, wie französische Menuets tanzen, oder gar allesamt Hexameter und horazische Oden schreiben.»

16. Ibid., p. 550,561.

17. Carl Schmitt, Politische Romantik, Munich/Leipzig, Duncker et Humboldt, 1919.

18. Johann Gottfried von Herder, Volklieder, p. 61-62. 